\title{
Correlation between Hypertension and SYNTAX Score in Patients with Chest Pain Admitted to Cardiology Department for Coronary Angiography
}

\author{
Ayman Abdulwahed Saif Mohammed, Xin Lin, Ekhlas Al hashedi, Runmin Sun, Jing Yu* \\ Department of Cardiology, Lanzhou University Second Hospital, Lanzhou, China \\ Email: *yujing2304@126.com
}

How to cite this paper: Saif Mohammed, A.A., Lin, X., Al hashedi, E., Sun, R.M. and Yu, J. (2021) Correlation between Hypertension and SYNTAX Score in Patients with Chest Pain Admitted to Cardiology Department for Coronary Angiography. World Journal of Cardiovascular Diseases, 11, 231-241.

https://doi.org/10.4236/wjcd.2021.114023

Received: March 5, 2021

Accepted: April 9, 2021

Published: April 12, 2021

Copyright () 2021 by author(s) and Scientific Research Publishing Inc. This work is licensed under the Creative Commons Attribution International License (CC BY 4.0).

http://creativecommons.org/licenses/by/4.0/ (c) (i) Open Access

\begin{abstract}
Background: Hypertension is associated with an increased risk of cardiovascular events, cardiovascular and all-cause mortality. However, the diagnostic ability of hypertension for the presence and severity of CAD (coronary artery disease) has not been elucidated. This study investigates the relationship between hypertension and CAD complexity using the SYNTAX score to determine hypertension's roles in coronary heart disease progression. Method: This is a prospective study that includes consecutive 410 adult patients at mean age (61 \pm 11 years) who are admitted to Cardiology Department and undergo invasive coronary angiography $(\mathrm{CAG})$ where a significant coronary lesion (SCL) is defined as stenosis $\geq 50 \%$ in vessel diameter $\geq 1.5 \mathrm{~mm}$. The SYNTAX scores were calculated using the SYNTAX score algorithm. Results: The mean rank of SYNTAX score was significantly higher among hypertension than non-hypertension (mean rank: $279,184, \mathrm{p}=0.006$ ) groups. SYNTAX score was positively correlated with age (r: $0.263, \mathrm{p}<0.001)$ and LDL (correlation coefficient $0.102, \mathrm{p}=0.038$ ) but inversely with HDL ( $\mathrm{r}: 0.107, \mathrm{p}=$ 0.031 ), in multivariate linear regression age (regression coefficient $0.3, p<$ $0.001)$, male $(-4.4, \mathrm{p}=0.002)$, HDL $(-6.4, \mathrm{p}=0.002)$ were significant independent risk factors for SYNTAX score, in ordinal regression model aging (odd ratio: 1.08, p < 0.001), being a male (2.84, $\mathrm{p}=0.026)$, HDL $(0.05, \mathrm{p}<$ $0.001)$, BMI $(0.86, p=0.020)$ were significantly independent predictor of increase or decrease probability of falling in high syntax score group. Conclusion Hypertension affects the distribution of SYNTAX score among patients with and without hypertension, and the prevalence of significant coronary lesions was more frequent in hypertensive patients. Hypertension was not a predictor of significant or complex coronary artery lesion, but advanced age,
\end{abstract}


being a male, HDL, LDL and BMI are considered as independent risk factors for high SYNTAX score, Subsequently and the complexity of CAD. Therefore, when patients with CAD have these factors, we expect that the Patient's CAD complexity will be high.

\section{Keywords}

BP, CAD, CAG, Complex Coronary Artery Disease, SCL, Coronary Lesion

\section{Introduction}

Cardiovascular disease (CVD) is still the main cause of mortality in industrialized nations [1]. Elevated blood pressure is associated with an elevated risk of cardiovascular events, cardio-vascular and all-cause mortality. In the Global Burden of Disease study, high blood pressure (HTN) was the leading risk factor globally, accounting for 9.4 million deaths and 7\% of global disability-adjusted life years (DALYs) in 2010 [2]. HTN accounts for about $90 \%$ of the risk for myocardial infarction in both genders [3]. However, the diagnostic ability of HTN for complex and severe coronary artery disease (CAD) has not been clearly elucidated. Previous clinical trials and observational studies showed a strong association, an etiological association between blood pressure (BP) and coronary heart disease. A pathophysiology shows that elevated BP accelerates atherosclerotic plaques development, narrowing arterial lumen by invading and spreading along arterial walls, embolizing, and increasing infarction risk. [4]

Coronary angiography is essential to select the ideal strategy of coronary revascularization by accurate characterization of coronary vessels. Recently, the Synergy between percutaneous coronary intervention with TAXus and cardiac surgery (SYNTAX) score has provided a tremendous amount of interest because of its ability to risk stratification and distinguish outcomes of patients with CAD undergoing revascularization percutaneously (PCI) compared with bypass graft surgery (CABG) [5].

\section{SYNTAX Score}

The SYNTAX score is a coronary angiographic score system based on coronary lesion. Firstly introduced to grade the complex CAD [6], it was used as a tool to help decide in the study of coronary angiography between cardiac surgeons and interventional cardiologists [7]. For patients with elevated SYNTAX score means that coronary artery lesion is complex and more challenging to revascularize by PCI. SYNTAX score was proven to be effective in predicting the clinical outcome after non-emergent PCI in patients with left main and/or 3-vessel CAD. SYNTAX score is also able to stratify risk for adverse very long-term clinical outcomes in patients receiving drug-eluting stents [8]. Previous studies conclud that elevated SYNTAX score is able to predict higher rates of major cardio-vascular events [9], absence reflow in patients with myocardial infarction (ST-elevation 
type) and contrast-induced nephropathy treated with primary PCI [10]. The SYNTAX score has been categorized to identify patients at low $(\leq 22)$, intermediate (23 to 32), and high risk ( $\geq 33$ ) [11]. The complex coronary lesion is an advanced stage and the reports about the risk of complex coronary lesions are many, but the reports on the risk factors of complex CAD are few because no ideal method indicates the complex CAD. Therefore, a risk factor for the complex coronary lesion is also thought to be a risk factor for complex CAD. The SYNTAX score is an index of complex CAD and not an indicator of severe coronary atherosclerosis [12]. A $99 \%$ or $50 \%$ stenosis lesions are equated and evaluated in SYNTAX score equally in complexity upon revascularization by PCI or CAG. Clearly, a $99 \%$ stenotic lesion must be related with a higher score than $50 \%$ stenosis if the severity of coronary atherosclerosis is evaluated. However, it is still unknown whether each coronary risk factor is an independent risk factor for complex CAD. Previous studies about the correlations between risk of the HTN with significant and complexity of coronary arterial lesions (CAL) are few and controversial.

\section{Methods}

\subsection{Study Design}

The present study is a prospective study that includes consecutive adult patients with chest pain admitted for suspected CAD in the Cardiology Department, Lanzhou University second hospital, between July 2019 and May 2020, investigate the association between hypertension and coronary artery disease complexity using SYNTAX score.

\subsection{Study Population}

A total of 410 consecutive patients who underwent CAG were enrolled. The exclusion criteria were as follows: cases with recent acute myocardial infarction, previous PCI or CABG, renal impairment and diabetic mellitus. Clinical data (age, history of smoking, alcohol, hypertension, diabetes mellitus and chronic kidney disease were collected by interview and serum total cholesterol, lowdensity lipoprotein cholesterol (LDL), and high-density lipoprotein (HDL), renal function test were done during admission and were collected from electronic medical records. This study was conducted with approval from the Ethics Committee of Lanzhou University second hospital, and informed consent was obtained from all the patients.

\subsection{SYNTAX Score Calculation}

Coronary angiogram (CAG) remains the gold standard for CAD diagnosis, and all participants underwent standardized, invasive CAG in the cardiology department of Lanzhou University second hospital, China. Standard coronary angiography was performed through the radial artery. CAG was analyzed and evaluated by two experienced interventional cardiologists. The result of CAG pre- 
sented as normal (without atherosclerotic stenosis), the significant coronary lesion (SCL) defined as $\geq 50 \%$ diameter stenosis in vessel $\geq 1.5 \mathrm{~mm}$ and non-significant stenosis (NSCL) $<50 \%$ stenosis. Coronary lesions with a lumen diameter $>1.5 \mathrm{~mm}$ and $\geq 50 \%$ diameter stenosis were further analyzed by SYNTAX score. Every significant lesion was analyzed separately according to Syntax score's guidelines described in detailed elsewhere [13] [14], and the SYNTAX score was calculated using the online SYNTAX score calculator $<$ http://www.syntaxscore.com/ $>$. SYNTAX score was used to integrate particular weighted factor for the specific segment number of only significant ( $\geq 50 \%$ diameter stenosis in a coronary vessel $\geq 1.5 \mathrm{~mm}$ ) lesions location and considering 11 morphologic features of each lesion (e.g. total occlusion, bi-furcation \& tri-furcation, length, thrombus, tortuosity, calcification, aorto-ostial, dominance, number of a diseased segment and diffused of a lesion). The BARI-2D trail [9] reported that a higher SYNTAX score could predict major events of cardiovascular. In the present study, we classified the final score into three group low $(0-22)$, intermediate $(23-32)$, high $(\geq 33)$.

\subsection{Hypertension Diagnostic Criteria}

The participant was classified as hypertensive if a patient had a history of hypertension and currently intaking antihypertensive drugs, a systolic office blood pressure $\geq 140 \mathrm{~mm} \mathrm{Hg}$ and or a diastolic office blood pressure $\geq 90 \mathrm{~mm} \mathrm{Hg}$ or by valid ABPM when average BP of 24 -hour ( $\geq 130 / 80)$, day-time $(\geq 135 / 85 \mathrm{~mm}$ $\mathrm{Hg}$ ), night-time ( $\geq 120 / 70 \mathrm{~mm} \mathrm{Hg}$ ), and early morning ( $\geq 135 / 85 \mathrm{~mm} \mathrm{Hg}$ ) [15].

\subsection{Statistical Analysis}

The normal distribution of data was assessed by the Kolmogorov-Smirnov test. Continuous variables are expressed as the mean $\pm \mathrm{SD}$ or median with range, and categorical variables are expressed as numbers (\%). For comparisons, the Mann Whitney $\mathrm{U}$ test was used to compare the distribution of SYNTAX score across hypertensive and non-hypertensive groups and other categories (gender, smoker and alcoholic). For normally distributed data, a comparison between two independent populations was done using an independent t-test. Counting data were compared by Chi-square test. Spearman's correlation was used to measure the strength of the association of SYNTAX score with risk factors. Multivariate linear regression analysis was used to analyze patient characteristics to determine the SYNTAX score's independent risk factors as a continuous variable. The Ordinal regression model was used to assess the predictor risk factors (categorical variables entered as factors and continuous variables as covariates) of being with low, intermediate, high SYNTAX score. SPSS 25 was used and a value of $\mathrm{p}<0.05$ was accepted as statistically significant.

\section{Result}

After the considered inclusion and exclusion criteria had been applied, 410 patients were included in the study. The patients' mean age was $61 \pm 11$ years and 
$260(63 \%)$ were men, $150(37 \%)$ were women. 279 (68\%) were hypertensive, 176 (43\%) were a current smoker or recently stopped smoking, 123 (30\%) were alcoholic. According to the CAG result: 176 (43\%) were with at least one SCL, and 243 (57\%) were with NSCL or normal CAG. According to SYNTAX score divided into three subgroups: 363 (88\%) were mild, 22 (5.5\%) intermediate, 25 (6\%) high SYNTAX score. The mean age was higher in the SCL group (64 \pm 11 years) than NSCL; the difference was statistically significant $(\mathrm{p}<0.05)$. Male patients with SCL were more likely than female; the difference was statistically significant $(\mathrm{p}<0.05)$. SCL more frequently in patients with hypertension than non-hypertensive patients, the difference was statistically significant $(\mathrm{p}<0.05)$. Non-smoker patients tended to be in the Non-SCL group than the SCL group; the difference was statistically significant $(\mathrm{p}<0.05)$. There was no significant difference in alcoholic patients, total cholesterol, LDL, HDL, triglyceride, BMI between SCL and Non-SCL group (Table 1).

The SYNTAX score distribution across hypertension, gender, smoker categories was differently significant. The mean rank in the hypertension group was higher than non-hypertension (mean rank: $279,184, \mathrm{p}=0.006$ ). The mean rank in the male gender was higher than the female (mean rank: 220, 181, p < 0.001). The smoker's mean rank was high than non-smoker (mean rank: 221, 194, p = $0.011)$.

SYNTAX score was positively correlated with age (correlation coefficient $0.263, \mathrm{p}<0.001$ ) and LDL (correlation coefficient $0.102, \mathrm{p}=0.038$ ) but inversely with HDL (correlation coefficient $0.107, \mathrm{p}=0.031$ ) (Table 2).

Table 1. Baseline characters based on CAG result.

\begin{tabular}{|c|c|c|c|c|}
\hline Variable & SCL 176 (43\%) & NSCL 243 (57\%) & Total (410) & p-value \\
\hline Age (years) & $64 \pm 11$ & $59 \pm 10$ & $61 \pm 10$ & $0.000^{*}$ \\
\hline Male & $127(31)$ & $133(32)$ & $260(63)$ & $0.001^{*}$ \\
\hline HTN & $131(32)$ & $148(36)$ & $279(68)$ & $0.016^{*}$ \\
\hline Non-HTN & $45(11)$ & $86(21)$ & $131(32)$ & \\
\hline Non-smoker & $89(22)$ & $145(35)$ & $234(57)$ & $0.026^{*}$ \\
\hline Alcoholic & $53(13)$ & $70(17)$ & $123(30)$ & 0.965 \\
\hline $\mathrm{TCH}(\mathrm{mmol} / \mathrm{l})$ & $4 \pm 1.2$ & $3.8 \pm 0.9$ & $3.9 \pm 1$ & 0.118 \\
\hline LDL (mmol/l) & $2.4 \pm 0.9$ & $2.3 \pm 0.8$ & $2.4 \pm 0.9$ & 0.211 \\
\hline HDL (mmol/l) & $1.1 \pm 0.3$ & $1.1 \pm 0.3$ & $1.2 \pm 0.33$ & 0.237 \\
\hline $\mathrm{TG}(\mathrm{mmol} / \mathrm{l})$ & $1.9 \pm 1.2$ & $1.9 \pm 1.4$ & $1.9 \pm 1.4$ & 0.922 \\
\hline BMI $\left(\mathrm{kg} / \mathrm{m}^{2}\right)$ & $24 \pm 2.8$ & $24 \pm 3.1$ & $24 \pm 3$ & 0.870 \\
\hline
\end{tabular}

Data are shown as mean \pm standard deviation, number (\%). Significant lesions: at least one $\geq 50 \%$ coronary stenosis in vessels diameter $\geq 1.5 \mathrm{~mm}$; TCH: total cholesterol; LDL: Low-density lipoprotein; HDL: High-density lipoprotein; TG: triglyceride; BMI: Body Mass Index; HTN: hypertension CAG: coronary angiography. ${ }^{\star}$ Significant $\mathrm{p}$-value $<0.05$. 
Table 2. Correlation between SYNTAX score and continuous variables.

\begin{tabular}{ccc}
\hline Variables & Correlation Coefficient & $\mathrm{p}$ \\
\hline Age (years) & $0.263^{* *}$ & $\mathbf{0 . 0 0 0}$ \\
TCH (mmol/l) & 0.068 & 0.171 \\
LDL (mmol/l) & $0.102^{*}$ & $\mathbf{0 . 0 3 8}$ \\
HDL (mmol/l) & $-0.107^{*}$ & $\mathbf{0 . 0 3 1}$ \\
TG (mmol/l) & 0.012 & 0.816 \\
BMI (kg/m $)$ & -0.007 & 0.884 \\
\hline
\end{tabular}

**: The correlation was significant at 0.01 level; * Correlation was significant at the 0.05 level: TCH: total cholesterol; LDL: Low-density lipoprotein; HDL: High-density lipoprotein; TG: triglyceride; BMI: Body Mass Index.

The results of multivariate linear regression analysis of risk factors for the SYNTAX score are shown in Table 3, aging, being a male, HDL were identified as significant independent risk factors (age: multiple regression coefficient $0.3, \mathrm{p}$ $<0.001$ ); male: $-4.4, \mathrm{p}=0.002$; HDL: $-6.4, \mathrm{p}=0.002)$. However, hypertension, total cholesterol, LDL, triglyceride, smoking, alcohol and BMI were not risking factors.

The ordinal regression model showed that aging (odd ratio: 1.08, $\mathrm{p}<0.001$ ), being a male (2.84, $\mathrm{p}=0.026)$, $\operatorname{HDL}(0.05, \mathrm{p}<0.001)$, BMI $(0.86, \mathrm{p}=0.020)$ were significant predictors of falling in low, intermediate, high SYNTAX score groups. However, hypertension, total cholesterol, LDL, triglyceride, smoking and alcohol were not significant predictors (Table 4).

\section{Discussion}

HTN is a modifiable risk factor for all clinical manifestations of CAD. The pathophysiological process of $\mathrm{BP}$ as a risk factor for CAD is complicated and includes the influence of BP as a physical force on the growth of the atherosclerotic plaque and the relationship between pulsating hemodynamic/arterial stiffness and coronary perfusion [15]. Therefore, secondary prevention of CAD is a significant task to improve the patient's clinical outcome with cardio-vascular disease. Conventional risk factors of CAD such as HTN, older age, male, dyslipidemia, BMI, and smoking have been related to more severe CAD [16]. A patient at a high-risk condition is anticipated to be accompanied by a severe form of the disease, so patients at high-risk CAD factor is suspected of having a severe form and complex CAD. Nevertheless, the evidence is still not that sufficient [6]. In this study, we investigated the association between hypertension (in addition to other common risk factors) and CAD complexity using the SYNTAX score to determine the roles of hypertension in the predicting coronary heart disease.

Our observational study's main findings are the statistically significant distribution difference of SYNTAX score among HTN and non-HTN groups, and the frequency of hypertensive patients among the significant coronary artery lesion 
group was higher than non-hypertensive. The incidence of hypertensive patients among the SCL subgroup was higher than the normal and NSCL subgroup. This result could be indicated that CAD in patients with hypertension was more complicated than that in patients without hypertension. We also found that older age, male gender, HDL, BMI were significant predictors of the SYNTAX score and high SYNTAX score group.

Table 3. Multivariate linear regression analysis of SYNTAX score and risk factors.

\begin{tabular}{cccc}
\hline Variables & Unstandardized (B) & $95 \%$ CI & $\mathrm{p}$ \\
\hline Age (years) & 0.277 & 0.17 to 0.39 & $0.000^{*}$ \\
Male gender & -4.4 & -7.3 to -1.6 & $\mathbf{0 . 0 0 2}$ \\
Hypertension & -1.5 & -3.9 to 0.8 & 0.202 \\
TCH (mmol/l) & 0.8 & -1.07 to 2.7 & 0.396 \\
LDL (mmol/l) & 1.7 & -0.29 to 3.7 & 0.094 \\
HDL (mmol/l) & -6.4 & -10.4 to -2.4 & $0.002^{*}$ \\
TG (mmol/l) & -0.14 & -1.08 to 0.8 & 0.766 \\
BMI (kg/m $\left.{ }^{2}\right)$ & -0.37 & -0.74 to 0.007 & 0.054 \\
Smoker & -1.6 & -4.7 to 1.5 & 0.298 \\
Alcoholic & 1.6 & -1.3 to 4.6 & 0.273 \\
\hline
\end{tabular}

TCH: total cholesterol; LDL: Low density lipoprotein; HDL: High density lipoprotein; TG: triglyceride; BMI: Body Mass Index. CI: confidence interval ${ }^{*}$ Significant $p$ value $<0.05$.

Table 4. Predictors of low, intermediate, and high SYNTAX scores.

\begin{tabular}{cccccccc}
\hline & OR & Estim. & Std.Erorr & Wald & $\mathrm{p}$ & \multicolumn{2}{c}{$95 \%$ CI } \\
\cline { 2 - 7 } & & & & & & lower & upper \\
\hline Age (years) & 1.08 & 0.078 & 0.02 & 15.368 & $0.000^{*}$ & 0.039 & 0.117 \\
Male gender & 2.84 & 1.045 & 0.47 & 4.935 & $0.026^{*}$ & 0.123 & 1.96 \\
Hypertension & 1.29 & 0.254 & 0.432 & 0.346 & 0.557 & -0.593 & 1.1 \\
TCH (mmol/l) & 1.59 & 0.465 & 0.237 & 3.829 & 0.050 & -0.001 & 0.930 \\
LDL (mmol/l) & 1.51 & 0.415 & 0.265 & 2.541 & 0.117 & -0105 & 0.935 \\
HDL (mmol/l) & 0.05 & -2.977 & 0.829 & 12.887 & $0.000^{*}$ & -4.603 & -1.352 \\
TG (mmol/l) & 0.89 & -0.112 & 0.148 & 0.571 & 0.450 & -0.403 & 0.179 \\
BMI (kg/m ${ }^{2}$ ) & 0.86 & -0.151 & 0.065 & 5.395 & $0.020^{*}$ & -0.279 & -0.024 \\
Smoker & 0.92 & -0.079 & 0.477 & 0.027 & 0.869 & -1.013 & 0.855 \\
Alcoholic & 1.25 & 0.227 & 0.459 & 0.245 & 0.621 & -0.673 & 1.128 \\
\hline
\end{tabular}

Ordinal regression: TCH: total cholesterol; LDL: Low-density lipoprotein; HDL: High-density lipoprotein; TG: triglyceride; BMI: Body Mass Index. CI: confidence interval; OR: odd ratio; Estim: Estimate ${ }^{*}$ Significant $\mathrm{p}$-value $<0.05$ 
As mentioned, whether hypertension is a risk factor of complex coronary atherosclerosis remains to be explained, and the evidence is still not that sufficient. Previous studies showed that the study selected 462 patients with CHD [17] showed that the mean SYNTAX score was higher in the hypertensive group than the non-hypertensive group, and HTN was independent predictors of complex coronary artery lesion. But on the other hand, Tanaka et al. [6] found that old age, being a male and having diabetes mellitus were significant and independent risk factors for the SYNTAX score, but hypertension was not a significant independent risk factor. Another study of 431 patients undergoing PCI [18] showed no significant association between the mean of SYNTAX score and hypertension. A study with 2163 patients [19] showed coexistence of hypertension and diabetes was associated with a negative synergistic effect on the SYNTAX score, but hypertension without diabetes was not associated with a high SYNTAX score. A. M. El Kersh et al. [20] studied the correlation between SYNTAX score and risk factors concluded that age, diabetes mellitus and smoking were significant independent risk factors of the complex CAD and high SYNTAX score, but the hypertension was not significant independent risk factors for the complexity of CAD. Our study found that the SYNTAX score distribution was different between hypertensive and non-hypertensive and the hypertensive patient associated with SCL. However, hypertension was not significant independent predictors for the complexity of CAD. This indicates that hypertension might affect the complex CAL but not an independent predictor of complex coronary artery lesion. This difference could be related to masked hypertension that failed to be diagnosed by office BP, so further studies are needed in the future and considered 24-hr ambulatory blood pressure in hypertension diagnosis for all subjects to detect masked hypertension and blood pressure variability. Also, the evaluation of coronary lesion depended on visual assessment and could be one of the limitations in detected complex lesions.

It is known that blood vessels endothelium function impaired, and arterial atherosclerosis progresses with getting older age [21]. Tanaka et al. [6] reported that advanced age is an independent risk factor of complex CAD [6]. This study also found that advanced age is positively correlated with SYNTAX score and an independent risk factor of significant and complex coronary lesion. It was known from a previous study that estrogen shows anti arteriosclerosis consequence and CAD of women progression occur after menopause and it is thought that complex CAD in male gender is more severe than in female gender because men take long time to progress coronary atherosclerosis than women. However, women with CAD are described to have a smaller diameter of coronary artery, as well as higher frequency of diffuse coronary stenosis is not suitable for PCI, coronary dissection and calcification than men [6] [22] [23] [24]. The fact that the development of CAD in women at an older age than in men may explain why male gender is a strong risk factor of CAD. In this study, we found that male gender was independent predictor of SCL and higher SYNTAX score. So, a male patient with CAD is suspected to be complex and needs more adjustment for other risk factors. 
Modifiable risk factors are important in the secondary prevention; previous studies already showed the high impact of these factors [25]. It is reported that abnormal level of lipid combined with HTN considered for more than $50 \%$ of attributable risks factors of CVD in population [26]. This study also found that HDL, total Cholesterol, LDL and BMI were also independent predictors of SCL and SYNTAX score. Also, we found that smoking and alcohol status were independent predictors for the SCL. The difference between risk factor result can be explained by: Firstly, different lifestyles in different ethnic groups, eating habits, cultural geographical differences, and other extrinsic factors; Secondly, the possibility that the lesion discovery occurs late due to silent myocardial ischemia; Thirdly, these risk factors for the SYNTAX score may cause diffused arterial atherosclerosis and gradually extended which form complex lesions (higher SYNTAX score), such as calcified, at bifurcated and long lesions for a longer period time. However, other factors may cause coronary atherosclerosis rapidly and locally extend before a lesion becomes complex. Lastly drugs such as statin or antihypertension could contribute.

\section{Study Limitations}

There are several limitations to this study: 1) the assessment of SYNTAX score depends on visual interpretation of lesion severity by clinical operators so the reproducibility of the SYNTAX score may limit its applicability in day-to-day clinical practice. 2) This study was an observational study at a single center. 3) Acute ST-elevation myocardial infarction was excluded because this was excluded as a subject of evaluation by the SYNTAX score. 4) We didn't demonstrate the effect of treating different modifiable risk factors on CAD lesion complexity. Further study in the future to examine this issue is required.

\section{Conclusion}

In terms of hypertension, the elevated blood pressure can be affecting the distribution of SYNTAX score among patients and the prevalence of significant coronary lesion more in hypertensive patients. In terms of coronary artery lesion risk factors, being an advanced age, being a male, HDL, LDL, BMI are considered independent predictors for high SYNTAX score and the complex CAD. Therefore, when patients with CAD have these factors, expectation of the complexity of CAD is present and the patient will be at high risk of complication and it is necessary to provide more medical care.

\section{Conflicts of Interest}

The authors declare no conflicts of interest regarding the publication of this paper.

\section{References}

[1] Shavelle, D.M. (2016) Almanac 2015: Coronary Artery Disease. Heart, 102, 492-499. 
https://doi.org/10.1136/heartjnl-2015-307761

[2] Lim, S.S., Vos, T., Flaxman, A.D., et al. (2012) A Comparative Risk Assessment of Burden of Disease and Injury Attributable to 67 Risk Factors and Risk Factor Clusters in 21 Regions, 1990-2010: A Systematic Analysis for the Global Burden of Disease Study 2010. The lancet, 380, 2224-2260.

https://doi.org/10.1016/S0140-6736(12)61766-8

[3] Yusuf, S., Hawken, S., Ôunpuu, S., et al. (2004) Effect of Potentially Modifiable Risk Factors Associated with Myocardial Infarction in 52 Countries (the INTERHEART Study): Case-Control Study. The Lancet (London, England), 364, 937-952. https://doi.org/10.1016/S0140-6736(04)17018-9

[4] Lawes, C.M.M., Bennett, D.A., Lewington, S. and Rodgers, A. (2002) Blood Pressure and Coronary Heart Disease: A Review of the Evidence. Seminars in Vascular Medicine, 2, 355-368. https://doi.org/10.1055/s-2002-36765

[5] Généreux, P., Palmerini, T., Caixeta, A., et al. (2011) SYNTAX Score Reproducibility and Variability between Interventional Cardiologists, Core Laboratory Technicians,and Quantitative Coronary Measurements. Circulation: Cardiovascular Interventions, 4, 553-561.

[6] Tanaka, T., Seto, S., Yamamoto, K., Kondo, M. and Otomo, T. (2013) An Assessment of Risk Factors for the Complexity of Coronary Artery Disease Using the SYNTAX Score. Cardiovascular Intervention and Therapeutics, 28, 16-21. https://doi.org/10.1007/s12928-012-0112-5

[7] Emren, S.V., Gediz, R.B., Şenöz, O., Karagöz, U., Şimşek, E.Ç. and Levent, F. (2019) "Decreased Heart Rate Recovery May Predict a High SYNTAX Score in Patients with Stable Coronary Artery Disease. Bosnian Journal of Basic Medical Sciences, 19, 109-115.

[8] Girasis, C., Garg, S., Räber, L., Sarno, G., et al. (2011) SYNTAX Score and Clinical SYNTAX Score as Predictors of Very Long-Term Clinical Outcomes in Patients Undergoing Percutaneous Coronary Interventions: A Substudy of SIRolimus-Eluting Stent Compared with pacliTAXel-Eluting Stent for Coronary Revascularization (SIRTAX) Trial. European Heart Journal, 32, 3115-3127. https://doi.org/10.1093/eurheartj/ehr369

[9] Ikeno, F., Brooks, M.M., Nakagawa, K., et al. (2017) SYNTAX Score and Long-Term Outcomes: The BARI-2D Trial. Journal of the American College of Cardiology, 69, 395-403.

[10] Uçar, H., Gür, M. Börekçi, A., et al. (2015) Relationship between Extent and Complexity of Coronary Artery Disease and Different Left Ventricular Geometric Patterns in Patients with Coronary Artery Disease and Hypertension. The Anatolian Journal of Cardiology, 15, 789-794. https://doi.org/10.5152/akd.2014.5747

[11] Serruys, P.W., Morice, M.-C., Kappetein, A.P., Feldman, T.E., et al. (2009) Percutaneous Coronary Intervention versus Coronary-Artery Bypass Grafting for Severe Coronary Artery Disease. The New England Journal of Medicine, 360, 961-972.

[12] Sianos, G., Morel, M., Kappetein, A.P., et al. (2005) The SYNTAX Score: An Angiographic Tool Grading the Complexity of Coronary Artery Disease. EuroIntervention, 1, 219-227.

[13] Yang, C.-H., Hsieh, M.-J., Chen, C.-C., et al. (2012) SYNTAX Score: An Independent Predictor of Long-Term Cardiac Mortality in Patients with Acute ST-Elevation Myocardial Infarction. Coronary Artery Disease, 23, 445-449. https://doi.org/10.1097/MCA.0b013e3283587835

[14] Serruys, P.W., Onuma, Y., Garg, S., et al. (2009) Assessment of the SYNTAX score 
in the Syntax study. EuroIntervention, 5, 50-56.

[15] Umemura, S., Arima, H., Arima, S., Asayama, K., Dohi, Y., Hirooka, Y., et al. (2019) The Japanese Society of Hypertension Guidelines for the Management of Hypertension (JSH 2019). Hypertension Research, 42, 1235-1481.

[16] Weber, T., Lang, I., Zweiker, R., et al. (2016) Hypertension and Coronary Artery Disease: Epidemiology, Physiology, Effects of Treatment, and Recommendations: A Joint Scientific Statement from the Austrian Society of Cardiology and the Austrian Society of Hypertension. Wiener klinische Wochenschrift, 128, 467-479. https://doi.org/10.1007/s00508-016-0998-5

[17] Wilson, P.W., D’Agostino, R.B., Levy, D., Belanger, A.M., Silbershatz, H. and Kannel, W.B. (1998) Prediction of Coronary Heart Disease Using Risk Factor Categories. Circulation, 97, 1837-1847. https://doi.org/10.1161/01.CIR.97.18.1837

[18] Zhang, J.X., Dong, H.Z., Chen, B.W., Cong, H.L. and Xu, J. (2016) Characteristics of Coronary Arterial Lesions in Patients with Coronary Heart Disease and Hypertension. SpringerPlus, 5, Article No. 1208. https://doi.org/10.1186/s40064-016-2828-7

[19] Salari, A., Mirbolook, F., Moladoust, H., Kheirkhah, J., Salari, A. and Etezadi, A. () "Predictive value of SYNTAX score on in-hospital outcomes after Percutaneous Coronary Intervention (PCI). Healthy Aging Research, 8, 3.

[20] Youn, Y.J., Lee, J.H., Lee, W., et al. (2012) ACC-i2 with TCT: Effect of Hypertension and Diabetes on High Syntax Score in Patients with Coronary. Journal of the American College of Cardiology, 59, E99. https://doi.org/10.1016/S0735-1097(12)60100-4

[21] El Kersh, A.M., Reda, A.A., El Hadad, M.G. and El-Sharnouby, K.H. (2018) Correlation between SYNTAX Score and Pattern of Risk Factors in Patients Referred for Coronary Angiography in Cardiology Department, Menoufia University. World Journal of Cardiovascular Diseases, 8, 431-439.

https://doi.org/10.4236/wjcd.2018.88042

[22] Celermajer, D.S., Sorensen, K.E., Spiegelhalter, D.J., Georgakopoulos, D., Robinson, J. and Deanfield, J.E. (1994) Aging Is Associated with Endothelial Dysfunction in Healthy Men Years before the Age-Related Decline in Women. Journal of the American College of Cardiology, 24, 471-476.

[23] Inoue, F., Yamaguchi, S., Ueshima, K., et al. (2010) Gender Differences in Coronary Plaque Characteristics in Patients with Stable Angina: A Virtual Histology Intravascular Ultrasound Study. Cardiovascular Intervention and Therapeutics, 25, 40-45. https://doi.org/10.1007/s12928-009-0009-0

[24] Nakamura, T., Ogita, M., Ako, J. and Momomura, S. (2010) Gender Differences of Plaque Characteristics in Elderly Patients with Stable Angina Pectoris: An Intravascular Ultrasonic Radiofrequency Data Analysis. International Journal of Vascular Medicine, 2010, Article ID: 134692. https://doi.org/10.1155/2010/134692

[25] Jacobs, A.K. (2015) Coronary Revascularization in Women in 2003. Circulation, 107, 375-377. https://doi.org/10.1161/01.CIR.0000048895.18974.6B

[26] Zengin, E., Bickel, C., Schnabel, R.B., Zeller, T. and Lackner, K. (2015) Risk Factors of Coronary Artery Disease in Secondary Prevention-Results from the AtheroGene-Study. PLoS ONE, 10, e0131434. https://doi.org/10.1371/journal.pone.0131434 